\title{
$t$-Pebbling and Extensions
}

\author{
David S. Herscovici* \\ Benjamin D. Hester ${ }^{\dagger}$ \\ Glenn H. Hurlbert ${ }^{\ddagger}$
}

November 2, 2018

\begin{abstract}
Graph pebbling is the study of moving discrete pebbles from certain initial distributions on the vertices of a graph to various target distributions via pebbling moves. A pebbling move removes two pebbles from a vertex and places one pebble on one of its neighbors (losing the other as a toll). For $t \geq 1$ the $t$-pebbling number of a graph is the minimum number of pebbles necessary so that from any initial distribution of them it is possible to move $t$ pebbles to any vertex.

We provide the best possible upper bound on the $t$-pebbling number of a diameter two graph, proving a conjecture of Curtis, et al., in the process. We also give a linear time (in the number of edges) algorithm to $t$-pebble such graphs, as well as a quartic time (in the number of vertices) algorithm to compute the pebbling number of such graphs, improving the best known result of Bekmetjev and Cusack.

Furthermore, we show that, for complete graphs, cycles, trees, and cubes, we can allow the target to be any distribution of $t$ pebbles without increasing the corresponding $t$-pebbling numbers; we conjecture that this behavior holds for all graphs.

Finally, we explore fractional and optimal fractional versions of pebbling, proving the fractional pebbling number conjecture of Hurlbert and using linear optimization to reveal results on the optimal fractional pebbling number of vertex-transitive graphs.
\end{abstract}

*Department of Mathematics and Computer Science, CL-AC3, Quinnipiac University, 275 Mount Carmel Avenue, Hamden, CT 06518, David.Herscovici@quinnipiac . edu

${ }^{\dagger}$ Department of Mathematics and Statistics, Arizona State University, Tempe, AZ, 85287, Ben jamin. Hester@asu . edu

${ }^{\ddagger}$ Department of Mathematics and Statistics, Arizona State University, Tempe, AZ, 85287, hurlbert@asu . edu 


\section{Introduction}

For a graph $G=(V, E)$, a function $D: V \rightarrow \mathbb{N}$ is called a distribution on the vertices of $G$, or a distribution on $G$. We usually imagine that $D(v)$ pebbles are placed on $v$ for each vertex $v \in V$. Let $|D|$ denote the size of $D$, i.e. $|D|=\sum_{v \in V} D(v)$. For two distributions $D$ and $D^{\prime}$ on $G$, we say that $D$ contains $D^{\prime}$ if $D^{\prime}(v) \leq D(v)$ for all $v \in V$. We write $\operatorname{diam}(G)$ for the diameter of $G$ and $\operatorname{dist}(v, w)$ for the distance

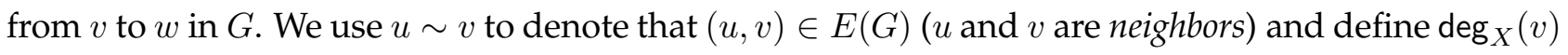
to be the number of neighbors of $v$ in the set $X$. In addition, we write $v \sim X$ when $\operatorname{deg}_{X}(v) \geq 1$. Here $n$ will represent the number of vertices of $G$. The following definition stipulates how pebbles can be transferred from one vertex to another.

Definition: A pebbling move in $G$ takes two pebbles from a vertex $v \in V$, which contains at least two pebbles, and places a pebble on a neighbor of $v$. Thus, one pebble is lost.

For two distributions $D$ and $D^{\prime}$, we say that $D^{\prime}$ is reachable from $D$ if there is some (possibly empty) sequence of pebbling moves beginning with $D$ and resulting in a distribution which contains $D^{\prime}$. We say that the cost of such a sequence is the sum of the number of pebbles lost along the way and the number of pebbles placed onto $D^{\prime}$.

Definitions: For an integer $t \geq 1$, we say a distribution $D$ on a graph $G$ is $t$-fold solvable if every distribution with $t$ pebbles on a single vertex is reachable from $D$. If $t=1$ we say the distribution is solvable; otherwise it is unsolvable. The t-pebbling number of a graph $G$, denoted $\pi_{t}(G)$, is the smallest integer $k$ such that every distribution $D$ with $|D| \geq k$ is $t$-fold solvable. The pebbling number of $G$ is $\pi_{1}(G)$, and we denote it $\pi(G)$.

In a sequence of pebbling moves, a distribution we are attempting to reach is called a target and a vertex we are attempting to reach is called a target vertex or a root. For a root $\mathbf{r}$, the quantity $\pi_{t}(G, \mathbf{r})$ is the smallest integer $k$ such that the distribution with $t$ pebbles on $\mathbf{r}$ and 0 pebbles on every other vertex is reachable from every distribution $D$ with $|D| \geq k$. 
Suppose that, instead of considering all possible distributions of a given size, we desire the smallest $t$-fold solvable distribution. In this spirit, we give the definition of the optimal $t$-pebbling number of a graph.

Definition: For an integer $t \geq 1$, the optimal t-pebbling number of a graph $G$, denoted $\pi_{t}^{*}(G)$, is the smallest integer $k$ such that there exists a $t$-fold solvable distribution $D$ of pebbles on $V$ with $|D|=k$. The optimal pebbling number of $G$ is $\pi_{1}^{*}(G)$, and we denote it $\pi^{*}(G)$.

We now outline the remainder of the paper. The main result is Theorem 2.7, which provides the best possible upper bound $\pi_{t}(G) \leq \pi(G)+4 t-4$ when $G$ has diameter 2 , and proves a conjecture of [7] as a corollary. Furthermore, we obtain an algorithm that places $t$ pebbles on any root from a distribution of $\pi(G)+4 t-4$ pebbles on the $n$ vertices of such $G$, and that runs in at most $6 n+$ $\min \{3 t, m\}$ steps, where $G$ has $m$ edges. We use this to build another algorithm that calculates $\pi(G)$ (distinguishing the two cases $n$ and $n+1$ ) of such $G$ in $O\left(n^{4}\right)$ time, besting the work of [1] when $m \gg n$. Motivated by prior work of Bukh [3] and Postle et al. [12], we consider graphs of larger diameter at the end of Section 2. In particular, we address a conjecture from [12] and show that any upper bound on the maximum pebbling numbers of such graphs must be at least exponential in the diameter.

In Section 3, we consider extensions and generalizations of $t$-pebbling numbers. In the definition of $\pi_{t}(G)$ the target is any distribution of $t$ pebbles that all sit on the same vertex. In the definition of $\pi(G, t)$ the target is any distribution of $t$ pebbles whatsoever. Necessarily, $\pi(G, t) \geq \pi_{t}(G)$. We prove that $\pi(G, t)=\pi_{t}(G)$ when $G$ is a complete graph, cycle, tree, or cube, and we conjecture that equality holds for all $G$. In addition, we prove a conjecture of [9] (Theorem 3.12), which states that the fractional pebbling number of a graph $G$ is $2^{\operatorname{diam}(G)}$. In exploring these extensions, we are naturally led to consider optimal fractional pebbling numbers, which provide some combinatorial insight into the fractional world of pebbling. In addressing fractional optimal pebbling numbers, we see that 
they can be found by appealing to linear optimization. In the end of the paper, we exploit this fact to discover results concerning optimal fractional pebbling numbers of certain graphs and classes of graphs.

\section{$2 t$-Pebbling}

In Section 2.1, we describe prior work on pebbling in trees and cycles. In Section 2.2, we prove a bound on $\pi_{t}(G, \mathbf{r})$ which will be useful in subsequent sections. In Section 2.3, we prove our main result, which provides an upper bound on the $t$-pebbling number of a graph which has diameter 2 . In Section 2.5, we address graphs of larger diameter by strengthening the known asymptotic lower bound on the value $\pi(n, d)$, the maximum pebbling number of an $n$-vertex graph with diameter $d$.

\subsection{Trees and Cycles}

To find the pebbling number of a vertex $\mathbf{r}$ in a tree $T$, Chung [5] defined $T_{\mathbf{r}}^{*}$ as the directed graph in which all edges in $T$ are directed toward $\mathbf{r}$. She then described path partitions and maximal path partitions in the tree $T_{r}^{*}$, which we generalize to describe path partitions in the undirected tree $T$ as well.

Definition (Chung [5]): A path partition of a undirected tree $T$ or of a tree $T_{\mathbf{r}}^{*}$ in which all edges are directed toward the vertex $\mathbf{r}$ is a partition of the edges of the tree into sets in such a way that the edges in each set in the partition form a path in $T$, or a path directed toward $\mathbf{r}$ in $T_{\mathbf{r}}^{*}$. The path-size sequence of a path partition is the sequence of lengths of the paths in nonincreasing order, $a_{1} \geq a_{2} \geq \cdots \geq a_{k}$. A maximal path partition in $T$ or in $T_{\mathbf{r}}^{*}$ is a path partition whose path-size sequence is lexicographically greatest.

Chung found $\pi_{t}(T, \mathbf{r})$, and Bunde et al. [4] gave $\pi_{t}(T)$. We present these results as Theorem 2.1] 
Theorem 2.1 (Chung [5]; Bunde et al. [4]) If $\mathbf{r}$ is a vertex in a tree $T$, then $\pi_{t}(T, \mathbf{r})$ is given by

$$
\pi_{t}(T, \mathbf{r})=2^{a_{1}} t+2^{a_{2}}+\ldots+2^{a_{k}}-k+1
$$

where $a_{1}, a_{2}, \ldots, a_{k}$ is the path-size sequence of a maximal path partition of $T_{\mathbf{r}}^{*}$. Then $\pi_{t}(T)=\pi_{t}(T, \mathbf{r})$, where $\mathbf{r}$ is chosen to be the root corresponding to a maximal path partition of $T$.

Although it was certainly clear from Chung's work, it appears that no one has formally stated and proved that moving a pebble to $\mathbf{r}$ costs at most $2^{a_{1}}$ pebbles from the rest of the graph. We prove this now.

Proposition 2.2 Let $\mathbf{r}$ be any vertex in the tree $T$ and suppose $D$ is a distribution on $T$ from which $t$ pebbles can be moved to $\mathbf{r}$. Then it is possible to move $t$ pebbles to $\mathbf{r}$ at a cost of at most $2^{a_{1}} t$ pebbles from the rest of the graph, where $a_{1}=\max _{v \in V(T)} \operatorname{dist}(\mathbf{r}, v)$. In particular, $t$ pebbles can be moved to any vertex at a cost of at most $2^{\operatorname{diam}(G)} t$ pebbles from the rest of the graph.

Proof: Let $S$ be a minimal sequence of pebbling moves that places $t$ pebbles on $\mathbf{r}$. For every $i \in$ $\left\{0,1, \ldots, a_{1}\right\}$ let $L_{i}=\{u \in V(G) \mid \operatorname{dist}(\mathbf{r}, u)=i\}$, so $L_{i}$ is the $i$ th level in the tree rooted at $\mathbf{r}$. For $i<a_{1}$ let $n_{i}$ denote the number of pebbling moves in $S$ from $L_{i+1}$ to $L_{i}$. Now $n_{0}=t$, and for larger $i$ we need at most $2 n_{i-1}$ moves onto $L_{i}$ to make $n_{i-1}$ moves onto $L_{i-1}$; therefore, by induction, we have $n_{i} \leq 2^{i} t$. Thus, the number of pebbling moves in $S$ is at most $\sum_{i=0}^{a_{1}-1} 2^{i} t=\left(2^{a_{1}}-1\right) t=2^{a_{1}} t-t$. Each such pebbling move results in the loss of a pebble. Thus, along with the $t$ pebbles that ends up on $\mathbf{r}$, at most $2^{a_{1}} t$ pebbles are removed from the rest of the graph.

The $t$-pebbling number $\pi_{t}\left(C_{n}\right)$ is given in [8]. We will refer to this result in Section 3 ,

Proposition 2.3 (Herscovici [8]) The t-pebbling number of a cycle is given by

$$
\begin{gathered}
\pi_{t}\left(C_{2 k}\right)=2^{k} \cdot t \\
\pi_{t}\left(C_{2 k+1}\right)=\frac{2^{k+2}-(-1)^{k}}{3}+2^{k}(t-1) .
\end{gathered}
$$




\subsection{A Distance-Based Bound}

In this section, we prove Theorem 2.4.

Theorem 2.4 Suppose $\mathbf{r}$ is a vertex in a graph $G$ with the property that $\operatorname{dist}(v, \mathbf{r}) \leq d$ for every vertex $v$ in $G$. Then

$$
\pi_{t}(G, \mathbf{r}) \leq \frac{2^{d}-1}{d}(n-1)+2^{d}(t-1)+1
$$

Furthermore, if there are at least $\frac{2^{d}-1}{d}(n-1)+1$ pebbles on the graph, moving a pebble to $\mathbf{r}$ costs at most $2^{d}$ pebbles from the rest of the graph.

Proof of Theorem 2.4 Let $T$ be a spanning tree of $G$ obtained by doing a breadth-first search from r. Since $T$ is a spanning subgraph of $G$, we have $\pi_{t}(G, \mathbf{r}) \leq \pi_{t}(T, \mathbf{r})$. Because $T$ was obtained by a breadth-first search from $\mathbf{r}$, we have $\operatorname{dist}_{G}(\mathbf{r}, v)=\operatorname{dist}_{T}(\mathbf{r}, v)$ for every vertex $v$ in $G$. Therefore, it suffices to show that (1) holds when $G=T$. Let $a_{1}, a_{2}, \ldots, a_{k}$ be the path-size sequence of a maximal path partition of $T_{\mathbf{r}}^{*}$. In particular, we have each $a_{i} \leq d$. Then we have

$$
\sum_{i=1}^{k} a_{i}=|E(T)|=|V(T)|-1=n-1
$$

since each edge in the tree is in exactly one part in the partition. From Theorem 2.1, we also have

$$
\pi_{t}(T, \mathbf{r})=2^{a_{1}} t+2^{a_{2}}+\ldots+2^{a_{k}}-k+1
$$

which, we can rewrite as

$$
\pi_{t}(T, r)=\sum_{i=1}^{k}\left(2^{a_{i}}-1\right)+2^{a_{1}}(t-1)+1=\sum_{i=1}^{k}\left[\left(\frac{2^{a_{i}}-1}{a_{i}}\right) a_{i}\right]+2^{a_{1}}(t-1)+1 .
$$

Now since each $a_{i}$ is at most $d$, we use the fact that $\left(2^{d}-1\right) / d$ is an increasing function on the positive integers to obtain

$$
\pi_{t}(T, \mathbf{r}) \leq \sum_{i=1}^{k}\left[\left(\frac{2^{d}-1}{d}\right) a_{i}\right]+2^{d}(t-1)+1=\left(\frac{2^{d}-1}{d}\right) \sum_{i=1}^{k} a_{i}+2^{d}(t-1)+1
$$


But from (2), we find

$$
\pi_{t}(G, \mathbf{r}) \leq \pi_{t}(T, \mathbf{r}) \leq \frac{2^{d}-1}{d}(n-1)+2^{d}(t-1)+1
$$

Finally, since each move is made along a directed edge in $T_{\mathbf{r}}^{*}$, by Proposition 2.2 , at most $2^{d}$ pebbles from the rest of the graph are consumed.

Curtis et al. proved Theorem 2.5 .

Theorem 2.5 (Curtis et al. [7]) For any integer $t \geq 1$, if $G$ is a graph with diameter 2 , then $\pi_{t}(G) \leq n+$ $7 t-6$.

The proof of Theorem 2.5 can be generalized to prove Theorem 2.6 .

Theorem 2.6 (Curtis et al. [7|) If $\mathbf{r}$ is a vertex in $G$ such that $\operatorname{dist}(\mathbf{r}, v) \leq 2$ for every vertex $v$ in $G$, then $\pi_{t}(G, \mathbf{r}) \leq n+7 t-6$

Using Theorem 2.4 with $d=2$ gives the bound $\pi_{t}(G, \mathbf{r}) \leq 1.5 n+4 t-4.5$. Thus, Theorem 2.4 represents an improved bound on that given by Theorem 2.6 when $t>\frac{n+3}{6}$. In Section 2.3, we further improve this bound when the diameter of the graph is 2 .

\subsection{Graphs of Diameter 2}

We prove Theorem 2.7, which gives a bound on the $t$-pebbling number of graphs with diameter 2 .

Theorem 2.7 If $G$ is a graph with diameter 2 then $\pi_{t}(G) \leq \pi(G)+4 t-4$.

Star graphs, denoted $K_{1, p}$, feature prominently in our proof, so we define them now.

Definition: If $p \geq 2$, the star on $p+1$ vertices, denoted $K_{1, p}$, is the graph whose vertex and edge sets are given by $V\left(K_{1, p}\right)=\left\{u, v_{1}, v_{2}, \ldots, v_{p}\right\}$ and $E\left(K_{1, p}\right)=\left\{\left(u, v_{i}\right): 1 \leq i \leq p\right\}$. We call $u$ the center of the star, and we call the $v_{i}$ 's its leaves. By abuse of notation, we identify the vertex set $V$ with the star $K_{1, p}$ if $|V|=p+1$ and the subgraph induced by $V$ contains $K_{1, p}$. 
To prove Theorem 2.7, we let $D$ be a distribution of $\pi(G)+4 t-4$ pebbles on $G$ for some $t \geq 2$ (there is nothing to show if $t=1$ ), and we show that $t$ pebbles can be moved to the vertex $\mathbf{r}$. We assume by induction that $\pi_{t-1}(G) \leq \pi(G)+4(t-1)-4=\pi(G)+4 t-8$. Therefore, if we could move a pebble to $\mathbf{r}$ at a cost of no more than four pebbles, we could use the remaining $\pi(G)+4 t-8$ pebbles to put $t-1$ additional pebbles on $\mathbf{r}$. We show that if putting a pebble on $\mathbf{r}$ requires using five pebbles, then $n+4 t-4$ pebbles are sufficient to put $t$ pebbles on $\mathbf{r}$. To do this, we note that if four pebbles are not sufficient to move a pebble onto $r$, this places certain constraints on $D$. Lemmas 2.8, 2.9, and 2.10 formalize this idea.

Lemma 2.8 Suppose $G$ is a graph with diameter 2 , and $D$ is a distribution on $G$ from which any sequence of pebbling moves that puts a pebble on the vertex $\mathbf{r}$ requires at least five pebbles. Then every vertex has at most three pebbles, and no vertex with two or three pebbles can be adjacent to $\mathbf{r}$.

Lemma 2.9 Suppose $G$ is a graph with diameter 2 , and $D$ is a distribution that satisfies the condition of Lemma 2.8 Let $v_{i}$ be a vertex with at least two pebbles. Then there is a vertex $w_{i}$ adjacent to both $v_{i}$ and $\mathbf{r}$. Furthermore, every such $w_{i}$ is unoccupied.

Lemma 2.10 Suppose $G$ is a graph with diameter 2 , and $D$ is a distribution that satisfies the condition of Lemma 2.8 Suppose further that $v_{i}$ and $v_{j}$ are distinct vertices which each have two or three pebbles. Then the vertices $w_{i}$ and $w_{j}$ from Lemma 2.9 are also distinct.

Proof of Lemmas 2.8, 2.9, and 2.10 Let $v_{i}$ be any vertex with at least two pebbles. We note that if $v_{i}$ were adjacent to $\mathbf{r}$ (or if $v_{i}=\mathbf{r}$ ), two pebbles would be sufficient to reach $\mathbf{r}$. Since $\operatorname{diam}(G)=2$, there is a vertex $w_{i}$ that is adjacent to both $v_{i}$ and to $\mathbf{r}$. Therefore, four pebbles on $v_{i}$ would be sufficient to reach $\mathbf{r}$ (completing the proof of Lemma 2.8), and if $w_{i}$ were occupied, two pebbles on $v_{i}$ and one pebble on $w_{i}$ would be sufficient to reach $\mathbf{r}$ (establishing Lemma 2.9). Finally, if any $w_{i}$ were adjacent 
to both $v_{i}$ and $v_{j}$ in Lemma 2.10, then we could move one pebble onto $w_{i}$ from $v_{i}$ and another from $v_{j}$, and from there we could move a pebble onto $\mathbf{r}$ at a total cost of four pebbles.

Before proving Theorem 2.7, we introduce the new concept of resolving a subgraph. Essentially, if $D$ is a distribution on a graph $G$ and $H$ is an edge subgraph of $G$ (i.e. $E(H) \subseteq E(G)$ ), then we define the distribution $D_{H}$ on $G$ by $D_{H}(v)=D(v)$ for $v \in H$ and 0 otherwise. Vaguely, for some root $\mathbf{r}$ of $G$, when we say to resolve $H$, we mean to place as many pebbles on $\mathbf{r}$ as possible from the distribution $D_{H}$. When the time comes, for certain subgraphs having particular distributions, we will remove ambiguity by describing the necessary pebbling steps in sufficient detail.

For example, let $H$ be the star $K_{1, p}$ with $p \geq 2$, having 3 pebbles on each of its leaves and at most 2 pebbles on its center. In this case, we resolve the star by, first, moving pebbles from some of its leaves through the center and onto $l$ other leaves $L$ so that every leaf has at most 4 pebbles and so that $l$ is maximized and, second, moving $l$ pebbles onto $\mathbf{r}$ from $L$ (which is possible because $\operatorname{diam}(G)=2$ ).

Lemma 2.11 Let $H$ be a star $K_{1, p}$ with $p \geq 2$, whose center vertex has $i \leq 2$ pebbles and whose leaves each have three pebbles. Then resolving $H$ puts $l=\lfloor(p+i) / 3\rfloor$ pebbles on $\mathbf{r}$. Moreover, for $l^{\prime}=(p+i) \bmod 3($ so that $p+i=3 l+l^{\prime}$ and $\left.0 \leq l^{\prime} \leq 2\right)$, there remain $l^{\prime}$ leaves with 3 pebbles each, and the number of leaves used in the resolution equals $3 l-i$.

Proof: For every three leaves we can move pebbles from two leaves to the center and then one pebble to the third. For every pebble already on the center we save a pebbling step from a leaf to the center. This uses $3 l-i$ outer vertices, so the number of unused outer vertices is $p-(3 l-i)=p+i-3 l=l^{\prime}$.

Notice that Lemma 2.11 does not hold for $p=1$. For this reason, our strategy for placing $t$ pebbles on $\mathbf{r}$ in $G$ will also use matchings between vertices having 3 pebbles. We resolve a matching $M$ such as this by, first, arbitrarily moving one pebble across each edge and, second, moving a pebble from each recipient vertex to $\mathbf{r}$. The number of pebbles that reach $\mathbf{r}$ equals the number $m$ of edges of $M$, and all $2 m$ vertices of $M$ are used in this resolution. 
We are now ready to prove Theorem 2.7

Proof of Theorem 2.7 As discussed above, we may assume the hypothesis of Lemma 2.8; otherwise induction suffices. Our proof is algorithmic. In the first stage we resolve a matching; in the second we iteratively resolve stars. By some careful counting arguments we will show that $t$ pebbles reach $\mathbf{r}$.

We begin by defining, for $0 \leq k \leq 3$, the set $V_{k}=\{v \mid D(v)=k\}$, with $n_{k}=\left|V_{k}\right|$. Let $M$ be a maximal matching in the subgraph $G\left[V_{3}\right]$ induced by the vertices of $V_{3}$, and denote its number of edges by $m$. Resolve $M$ and let $D^{\prime}$ be the resulting distribution. For $0 \leq k \leq 3$ define $V_{k}^{\prime}=\{v \in$ $\left.V-V(M) \mid D^{\prime}(v)=k\right\}$. Notice that $V_{3}^{\prime}$ is independent in $G$.

Next we initialize the sets $S_{k}=\emptyset$ for $0 \leq k \leq 2$ and $L=\emptyset$, and iterate the following steps. For a vertex $v$ define $d_{3}^{\prime}(v)=\operatorname{deg}_{V_{3}^{\prime}}(v)$. Now for any $k$ choose some $v \in V_{k}^{\prime}$ with $d_{3}^{\prime}(v) \geq 3-\lfloor k / 2\rfloor$, if one exists (necessarily $k \leq 2$ ), and let $S$ be the star consisting of the center $v$ and all its neighbors in $V_{3}^{\prime}$. Resolve $S$, put $v$ in $S_{k}$, add the leaves of $S$ to $L$, redefine the notation $D^{\prime}$ for the resulting distribution, and likewise redefine the sets $V_{k}^{\prime}$ accordingly. At some point the algorithm halts because no possibilities remain for choosing an appropriate center $v$. Write $s$ for the number of pebbles that the stars contribute to $r$.

Over the course of the algorithm, Lemma2.11 implies that

$$
m+s=m+\sum_{k=0}^{2} \sum_{v \in S_{k}} l(v)
$$

pebbles have reached $\mathbf{r}$, where $l(v)=\left\lfloor\left(d_{3}^{\prime}(v)+k\right) / 3\right\rfloor$, and that

$$
2 m+\sum_{k=0}^{2} \sum_{v \in S_{k}}(3 l(v)-k)=2 m+3 s-s_{1}-2 s_{2}
$$

vertices from $V_{3}$ were used to send pebbles in the process, where $s_{k}=\left|S_{k}\right|$. The proof will be complete when we show that $m+s \geq t$.

At this time set $U=V_{3}^{\prime}-L, W=\left\{v \mid \operatorname{deg}_{U}(v) \geq 2\right\}$, and $Y=\left\{v \mid \mathbf{r} \sim v \sim V_{3}\right\}$. We note that $u=|U|=n_{3}-2 m-3 s+s_{1}+2 s_{2}$ (by (4)) and $|W| \geq\left(\begin{array}{l}u \\ 2\end{array}\right) \geq u-1$ (since $\operatorname{diam}(G)=2$ and no more 
stars exist). Observe also that $V_{3} \cap Y=W \cap Y=\emptyset$ because of the cost 5 assumption. Hence, for $Z=V_{3} \cup W \cup Y$ we compute

$$
\begin{aligned}
z=|Z| & =\left|V_{3}\right|+|Y|+\left|W-V_{3}\right| \\
& \geq n_{3}+n_{3}+(u-1-m) \\
& =3 n_{3}-3 m-3 s+s_{1}+2 s_{2}-1,
\end{aligned}
$$

the appearance of $m$ coming from the fact that, if $v \in W \cap V_{3}$, then having two neighbors in $U$ when the algorithm halts means that $D^{\prime}(v)=0, v \notin \cup_{k=0}^{2} S_{k}$, and consequently that $v$ was a recipient in the resolution of $M$. The number of such vertices was exactly $m$. We also can calculate the number of pebbles originally on $Z$.

$$
\begin{aligned}
|D(Z)| & =\left|D\left(V_{3}\right)\right|+|D(Y)|+\left|D\left(W-V_{3}\right)\right| \\
& \leq 3 n_{3}+0+s_{1}+2 s_{2} \\
& \leq z+3 m+3 s+1 .
\end{aligned}
$$

To complete the analysis we set $X_{k}=V_{k}-Z(0 \leq k \leq 2)$ and $x_{k}=\left|X_{k}\right|$; then $n=z+x_{0}+x_{1}+x_{2}$. Now we have

$$
\begin{aligned}
z+x_{0}+x_{1}+x_{2}+4 t-4 & =n+4 t-4 \\
& \leq|D| \\
& =|D(Z)|+x_{1}+2 x_{2} \\
& \leq z+3 m+3 s+1+x_{1}+2 x_{2} .
\end{aligned}
$$

Combined with the fact that $x_{0} \geq x_{2}+1$ (since $X_{0} \supseteq\{\mathbf{r}\} \cup\left\{v \mid \mathbf{r} \sim v \sim X_{2}\right\}$ ), this implies that $4 t-4 \leq 3 m+3 s$, from which follows

$$
m+s \geq\left\lceil\frac{4 t-4}{3}\right\rceil
$$




$$
\begin{aligned}
& =(t-1)+\left\lceil\frac{t-1}{3}\right\rceil \\
& \geq t,
\end{aligned}
$$

since $t \geq 2$. This completes the proof.

Pachter, Snevily, and Voxman [11] found the pebbling number for graphs with diameter 2. In particular, they showed the following theorem.

Theorem 2.12 (Pachter et al. [11]) If $G$ is a graph with diameter 2 , then $\pi(G) \leq n+1$.

Putting Theorems 2.7 and 2.12 together, gives us Corollary 2.13, first conjectured in [7].

Corollary 2.13 For any integer $t \geq 1$, if $G$ is a graph with diameter 2 , then $\pi_{t}(G) \leq n+4 t-3$.

One might ask whether there are any diameter 2 graphs $G$ and any values of $t$ where the inequality in Theorem 2.7 is strict, i.e. $\pi_{t}(G)<\pi(G)+4 t-4$. Indeed there are. Proposition 2.14 shows that the difference can be as large as $\pi(G)-4$.

Proposition 2.14 For $n \geq 3$, let $G_{n}$ be the graph obtained by removing the edge $\left\{v_{1}, v_{2}\right\}$ from the complete graph $K_{n}$. Then for any $t \geq n-2$, we have $\pi_{t}\left(G_{n}\right)=4 t$.

Proof: We have $\pi_{t}(G) \geq 4 t$, since placing $4 t-1$ pebbles on $v_{2}$ creates a distribution from which $t$ pebbles cannot be moved to $v_{1}$, so we need to show $\pi_{t}(G) \leq 4 t$. We use induction on $n$, and later, induction on $t$ as well. The basis is $n=3$. Now $G_{3}$ is the path on the vertices $\left\{v_{1}, v_{3}, v_{2}\right\}$, in that order, so $\pi_{t}\left(G_{3}\right)=4 t$, as desired.

For $n>3$, we assume $\pi_{t^{\prime}}\left(G_{n-1}\right)=4 t^{\prime}$ whenever $t^{\prime} \geq n-3$. We also assume $v_{1}$ is the target (or $\left.v_{2}\right)$; otherwise, applying Theorem 2.4 with $d=1$ gives $\pi_{t}(G) \leq n+2 t-2 \leq 3 t$.

Let $D$ be a distribution of $4 t$ pebbles on $G_{n}$, and let $p_{i}=D\left(v_{i}\right)$. We assume without loss of generality that $p_{3} \geq p_{4} \geq \cdots \geq p_{n}$. The rest of our argument depends on the value of $p_{n}$. If $p_{n}=0$ we have $4 t$ pebbles on the vertices $\left\{v_{1}, v_{2}, \ldots, v_{n-1}\right\}$. These vertices induce a subgraph isomorphic 
to $G_{n-1}$, so by our inductive assumption, $4 t$ pebbles are sufficient to $t$-pebble $v_{1}$. If $p_{n}=1$, we note that some vertex has two pebbles, and since $v_{n}$ is adjacent to every other vertex, we can put a second pebble onto $v_{n}$, and from there, we can put a pebble on $v_{1}$. After that, the remaining $4 t-3$ pebbles on $\left\{v_{1}, v_{2}, \ldots, v_{n-1}\right\}$ suffice to put an additional $(t-1)$ pebbles on $v_{1}$, again by our inductive assumption.

Finally, if $p_{n} \geq 2$, we resort to induction on $t$. We note that each of the $(n-2)$ vertices in $\left\{v_{3}, v_{4}, \ldots, v_{n}\right\}$ has at least two pebbles and is adjacent to $v_{1}$. Therefore, at least $(n-2)$ pebbles can be moved to $v_{1}$. Thus, if $t=n-2$, we are done. Otherwise, $t \geq n-1$, so we move one pebble from $v_{n}$ to $v_{1}$. Since $t-1 \geq n-2$, we may assume by induction on $t$ that the remaining $4 t-2$ pebbles are sufficient to move $(t-1)$ additional pebbles onto $v_{1}$.

\subsection{Algorithmic Results for Diameter 2 Graphs}

We remark that, for $|D| \geq \pi(G)+4 t-4$, we can place $t$ pebbles on any root $\mathbf{r}$ in time that is linear in $n$ and $t$ : at most $6 n+3 t$ steps are required. To see this, if one can place a pebble on $\mathbf{r}$ with cost at most 4, a breadth-first search from $\mathbf{r}$ will reveal it in at most $n$ steps, since the only possibilities (besides already having a pebble on $\mathbf{r})$ are $(i)$ a path $(\mathbf{r}, u)$ with distribution $(0, \geq 2)$, (ii) a path $(\mathbf{r}, u, v)$ with distribution $(0,1, \geq 2)$, (iii) a path $(\mathbf{r}, u, v, w)$ with distribution $(0,1,1, \geq 2)$, (iv) a path $(\mathbf{r}, u, v)$ with distribution $(0,0, \geq 4)$, and $(v)$ a star $(\mathbf{r}, u, v, w)$ with center $u$ and distribution $(0,0, \geq 2, \geq 2)$. In fact, it is not difficult to see that all these solutions can be found and resolved in at most $n+3 t$ steps, since each resolution involves at most 3 edges and the breadth-first search does not need to be repeated.

If none of these possibilities exist, then our algorithm will place the required pebbles on $\mathbf{r}$ in at most $3 n$ steps when $t \geq 2$. Indeed, since the directed graph formed by orienting the edges of $G$ in the direction of pebbling can be seen to be acyclic (which is not necessarily the case above), the total number of edges used in the algorithm is at most $n$; consequently we can implement the algorithm so as to postpone the actual pebbling steps so that each such edge is traversed once. Thus we only 
need to count the number of steps required to find the matching and stars. It takes $n$ steps to sort the vertices into the appropriate $V_{k}$. Then a maximal matching in $G\left[V_{3}\right]$ can be found in at most $v_{3} / 2$ steps (we are fortunate not to need a maximum matching). Finally, if we search first for star centers with the most pebbles, then we don't repeat vertices in our search, so the total time to find all stars is at most $n-v_{3}$, making the number of steps for finding the matching and all stars at most $n$. Including sorting and resolution, we use at most $3 n$ steps.

As mentioned, in the proof of Theorem 2.7, we only used our algorithm when $t \geq 2$. Here we observe that it actually works with a slight modification when $t=1$ as well. The only difference is that we look not only for matching edges in $V_{3}$, but also for edges between $V_{2}$ and $V_{3}$. Hence if we have been unsuccessful to this point in placing a pebble on $\mathbf{r}$, we will show that, to avoid the contradiction that $|D|<\pi(G)$, we can find one final solution method in at most $2 n$ steps. To do so, define the sets $V_{k, d}=\{v \mid D(v)=k, \operatorname{dist}(v, \mathbf{r})=d\}$, for which we know that $0 \leq d \leq 2$ and $0 \leq k<2^{d}$ (so that $V_{k}=V_{k, 2}$ for $k \in\{2,3\}$ ). In addition, we know that the neighbors of $V_{2} \cup V_{3}$ that lie in $V_{0,1}$ are distinct, and that the common neighbors of pairs of points $(u, v) \in V_{3} \times\left(V_{2} \cup V_{3}\right)$ lie in $V_{0,2}$ and are distinct also. Thus, in order that $|D| \geq n$ we must have $\left|V_{2}\right|+2\left|V_{3}\right| \geq\left|V_{0,1}\right|+\left|V_{0,2}\right|+1$; that is, there must be enough extra pebbles from $V_{2} \cup V_{3}$ to compensate for the lack of pebbles on $V_{0}$, including $\mathbf{r}$. In particular, this implies that $\left|V_{3}\right|>\left|V_{0,2}\right| \geq\left(\begin{array}{c}\left|V_{3}\right| \\ 2\end{array}\right)+\left|V_{3}\right|\left|V_{2}\right|$, which means that $\left|V_{3}\right| \in\{1,2\},\left|V_{2}\right|=0$, $\left|V_{0,2}\right|=\left(\begin{array}{c}\left|V_{3}\right| \\ 2\end{array}\right)$, and $\pi(G) \leq|D|=n$. Since we know that it is possible to place a pebble on $\mathbf{r}$, and we know it cannot begin by moving anything from $V_{3}$ to $V_{0,1}$, it must arise from moving pebbles from $S=V_{3} \cup V_{0,2}$ along a path through $V_{1}$ to r. Such a path $P$ can be found from a breadth-first search from $\mathbf{r}$ in $G\left[V-V_{0,1}\right]$; whichever vertex $v$ from $S$ is found first determines whether to move directly along $P$ from $v \in V_{3}$ or first to move 2 pebbles to $v \in V_{0,2}$ and then on to $P$. Finding and pebbling along $P$ takes at most $2 n$ steps.

We mention finally that if $3 t>m=m(G)$, the number of edges of $G$, we can perform the same 
trick in the first stage above as in the second, namely, that we postpone the actual resolution until the end, using at most $m$ steps instead of $3 t$. We record this in the following theorem.

Theorem 2.15 If $G$ is a graph with $n$ vertices, $m$ edges, and diameter 2 , and $D$ is a distribution of size $\pi(G)+4 t-4$, then $t$ pebbles can be placed on any root $\mathbf{r}$ in at most $6 n+\min \{3 t, m\}$ steps.

The only other algorithmic results known for diameter two graphs are found in [1]. There the authors consider the case when $t=1$ and $|D|<\pi(G)$, and present algorithms that determine the solvability of $D$ in polynomial time on graphs of constant bounded connectivity, among other cases. Their algorithm uses the characterization found in [6] (see also [2]) for diameter 2 graphs with pebbling number $n+1$, as opposed to $n$. They also use this characterization to give an $O\left(n^{3} m\right)$ algorithm for recognizing the difference. Here we use the $t=1$ portion of our algorithm to produce an $O\left(n^{4}\right)$ algorithm that recognizes the difference, which improves their result when $m \gg n$.

Theorem 2.16 If $G$ is a graph with $n$ vertices and diameter 2 , then it can be determined whether $\pi(G)=n$ or $n+1$ in $O\left(n^{4}\right)$ time.

Proof: We note that $\pi(G)=n+1$ if and only if there is a distribution $D$ of $n$ pebbles that cannot reach some r. For such a $D$ we have argued that it must have $\left|V_{3}\right| \in\{1,2\}$, along with the other conditions mentioned above. We use breadth-first search to determine distances from $\mathbf{r}$ and, for each $i \in\{1,2\}$ we pick $i$ vertices at distance 2 from $\mathbf{r}$ each of which has a unique common neighbor with $\mathbf{r}$, determining both $V_{3}$ and $V_{0,1}$ (such available choices can be filtered during the breadth-first search). We ignore the choice unless each chosen vertex has a unique common neighbor with $\mathbf{r}$, determining $V_{0,1}$, and when $i=2$ the pair in $V_{3}$ has a unique common neighbor, determining $V_{0,2}$. Now $\pi(G)=n$ if and only if there is a path from $V_{3} \cup V_{0,2}$ to $\mathbf{r}$ in $G\left[V-V_{3}-V_{0}\right]$. The total time required to complete these constructions and checks is at most $n+\left(n+n^{3} / 2\right) n=n^{4} / 2+n^{2}+n$. 


\subsection{Graphs of Larger Diameter}

It would be interesting to expand our methods with graphs of diameter 2 to graphs with larger diameter. Postle, Streib, and Yerger [12] announced Theorem[2.17, strengthening a result of Bukh [3].

Theorem 2.17 (Postle et al. [12]) If $G$ is a graph with diameter 3 , then $\pi(G) \leq 1.5 n+2$.

Let $\pi(n, d)$ denote the maximum pebbling number of an $n$-vertex graph which has diameter $d$. Bukh proved Theorem 2.18 ,

Theorem 2.18 (Bukh [3]) There are constants $c, N, D$ such that, for all $n>N$ and $d>D$, we have $\pi(n, d) \geq\left(\frac{{ }_{2}\left\lceil\frac{d}{2}\right\rceil-1}{\left\lceil\frac{d}{2}\right\rceil}\right) n+c$.

Postle et al. conjectured the following asymptotic upper bound on $\pi(n, d)$.

Conjecture 2.19 (Postle et al. [12]) There are constants $C, N$ and a function $f$ on the positive integers such that, for all $n>N$ we have $\pi(n, d) \leq\left(\frac{{ }_{2}\left\lceil\frac{d}{2}\right\rceil-1}{\left\lceil\frac{d}{2}\right\rceil}\right) n+C f(d)$.

We show that if Conjecture 2.19 holds, then $f(d)$ is at least exponential in $d$, for all $n$. We do this by creating $n$-vertex graphs of diameter $d$ that have large pebbling numbers for all $n$ and $d$. Given positive integers $n$ and $d$, we build the graph $G_{n, d}$ as follows.

If $d=2 k$, choose a vertex $v$ and build $\left\lfloor\frac{n-1}{k}\right\rfloor$ paths of length $k$ beginning at $v$ which are disjoint (except of course at $v$ ). If the number of vertices at this point is smaller than $n$, add one more path of length $n-k\left\lfloor\frac{n-1}{k}\right\rfloor-1$ which begins at $v$ and is disjoint from the rest of the graph. This is the graph $G_{n, 2 k}$.

If $d=2 k+1$, build a clique $K_{m}$, where $m=\left\lfloor\frac{n}{k+1}\right\rfloor$. From each vertex in this $K_{m}$, build a path of length $k$ which is disjoint from the rest of the graph. If the number of vertices at this point is smaller than $n$, choose any $v \in V\left(K_{m}\right)$ and add one more path of length $n-m(k+1)$ which begins at $v$ and 
is disjoint from the rest of the graph. This is the graph $G_{n, 2 k+1}$.

It is easy to check that $G_{n, 2 k}$ and $G_{n, 2 k+1}$ have $n$ vertices and diameters $2 k$ and $2 k+1$, respectively.

Proposition $2.20 \pi\left(G_{n, d}\right) \geq\left(\frac{2^{\left\lceil\frac{d}{2}\right\rceil}-1}{\left\lceil\frac{d}{2}\right\rceil}\right) n+\left(2^{d}-3\left(2^{\left\lceil\frac{d}{2}\right\rceil}-1\right)\right)$ for all $n$ and $d$. In particular, if Conjecture 2.19 holds, then $f(d) \geq c 2^{d}$ for some $c$ and all large enough $d$.

Proof: If $d=2 k$ for some $k$, then $G_{n, d}$ is a tree. In a maximal path partition, there is one path of length $2 k$ and there are $\left\lfloor\frac{n-1}{k}\right\rfloor-2$ paths of length $k$. Thus, from Theorem 2.1, we have

$$
\begin{aligned}
\pi\left(G_{n, d}\right) & \geq 2^{2 k}+2^{k}\left(\left\lfloor\frac{n-1}{k}\right\rfloor-2\right)-\left(\left\lfloor\frac{n-1}{k}\right\rfloor-1\right)+1 \\
& =\left(2^{k}-1\right)\left(\left\lfloor\frac{n-1}{k}\right\rfloor\right)+\left(2^{2 k}-2^{k+1}+2\right) \\
& \geq\left(2^{k}-1\right)\left(\frac{n}{k}-1\right)+\left(2^{2 k}-2^{k+1}+2\right) \\
& =\left(\frac{2^{\left\lceil\frac{d}{2}\right\rceil}-1}{\left\lceil\frac{d}{2}\right\rceil}\right) n+\left(2^{d}-3\left(2^{\left\lceil\frac{d}{2}\right\rceil}-1\right)\right) .
\end{aligned}
$$

If $d=2 k+1$ for some $k$, we build an unsolvable distribution $D$ on $G_{n, d}$. Let the root $\mathbf{r}$ be any leaf which is the endpoint of a maximum induced path $P$. Place $2^{2 k+1}-1$ pebbles on the other endpoint of $P$. Now, for every leaf vertex (disjoint from $P$ ) that is distance $k$ from $K_{m}$, place $2^{k+1}-1$ pebbles. There are $\left\lfloor\frac{n}{k+1}\right\rfloor-2$ such vertices. It is easy to verify that $D$ cannot send a pebble to $\mathbf{r}$. Thus, since $\pi\left(G_{n, d}\right) \geq|D|+1$

$$
\begin{aligned}
\pi\left(G_{n, d}\right) & \geq 2^{2 k+1}+\left(2^{k+1}-1\right)\left(\left\lfloor\frac{n}{k+1}\right\rfloor-2\right) \\
& =\left(2^{k+1}-1\right)\left(\left\lfloor\frac{n}{k+1}\right\rfloor\right)+\left(2^{2 k+1}-2^{k+2}+2\right) \\
& \geq\left(2^{k+1}-1\right)\left(\frac{n}{k+1}-1\right)+\left(2^{2 k+1}-2^{k+2}+2\right) \\
& =\left(\frac{2^{\left\lceil\frac{d}{2}\right\rceil}-1}{\left\lceil\frac{d}{2}\right\rceil}\right) n+\left(2^{d}-3\left(2^{\left\lceil\frac{d}{2}\right\rceil}-1\right)\right),
\end{aligned}
$$

as desired.

Conjecture2.19 would follow from Conjecture 2.21.

Conjecture 2.21 Let $G$ be any n-vertex graph with diameter $d$. Then $\pi(G) \leq \pi\left(G_{n, d}\right)$. 


\section{Extensions}

In Section 3.1, we consider how many pebbles are required to reach an arbitrary target distribution with $t$ pebbles. We conjecture an equality which would relate $\pi_{t}(G)$ to a more general pebbling invariant on a graph. The truth of the equality would simplify the process of obtaining general results about achieving arbitrary target distributions on graphs. In Section 3.2, we discuss how the $t$-pebbling number of a graph increases as $t$ increases. This naturally leads to the discussion of the fractional analogue of the pebbling number of a graph. We show that this value depends only on $\operatorname{diam}(G)$. In Section 3.3, we analyze the continuous version of optimal pebbling and present the corresponding linear optimization problem.

\subsection{Arbitrary Target Distributions with t Pebbles}

The following definition generalizes the definition of the $t$-pebbling number of a graph.

Definition: We define $\pi(G, t)$ as the smallest number of pebbles such that any target distribution $D$ with $|D|=t$ is reachable from every distribution $D^{\prime}$ with $\left|D^{\prime}\right| \geq \pi(G, t)$.

Clearly if we can reach any distribution with $t$ pebbles starting from $D$, we can reach any distribution with $t$ pebbles on a single vertex. Therefore, $\pi_{t}(G) \leq \pi(G, t)$ for every positive integer $t$. Conversely, it seems reasonable to believe that if we have a distribution of pebbles from which we can put $t$ pebbles on any single vertex of $G$, then any other distribution of $t$ pebbles is likewise reachable. For example, if we can put two pebbles either on the vertex $x$ or the vertex $y$, then we should be able to put one pebble each on $x$ and $y$. This suggests the following conjecture.

Conjecture 3.1 For every graph $G$ and every positive integer $t$, we have $\pi(G, t)=\pi_{t}(G)$.

One might be interested in a more general target distribution as a stepping stone to some goal. Having the equality from Conjecture 3.1 as a tool could greatly simplify the necessary analysis. We prove 
this conjecture for some common graphs. We start with two lemmas.

Lemma 3.2 Suppose $G$ is a graph with the property that, for some $t$, whenever $\pi_{t+1}(G)$ pebbles are on $G$, one pebble can be moved to any vertex at a cost of at most $\pi_{t+1}(G)-\pi(G, t)$ pebbles. Then $\pi(G, t+1)=\pi_{t+1}(G)$.

Proof: Let $D$ be a distribution on $G$ with $t+1$ pebbles. Given a distribution of $\pi_{t+1}(G)$ pebbles on $G$, choose one occupied vertex $v$ in $D$, and spend $\pi_{t+1}(G)-\pi(G, t)$ pebbles to move a pebble to $v$. The remaining $\pi(G, t)$ pebbles can be used to move $t$ additional pebbles to fill out the rest of $D$.

Lemma 3.3 Suppose $G$ is a graph with the property that for every $t$, if $\pi_{t+1}(G)$ pebbles are on $G$, one pebble can be moved to any vertex at a cost of at most $\pi_{t+1}(G)-\pi_{t}(G)$ pebbles. Then $\pi(G, t)=\pi_{t}(G)$ for all $t$.

Proof: We use induction on $t$. When $t=1$ we have $\pi_{1}(G)=\pi(G, 1)=\pi(G)$ since the target distributions are the same in either case. For larger $t$, if $\pi_{t}(G)=\pi(G, t)$, then $\pi_{t+1}(G)-\pi_{t}(G)=$ $\pi_{t+1}(G)-\pi(G, t)$, so by Lemma 3.2, $\pi_{t+1}(G)=\pi(G, t+1)$.

Theorems 3.4 and 3.5 gives some classes of graphs for which Conjecture 3.1 holds.

Theorem 3.4 Let $G$ be any graph such that $\pi(G)=2^{\operatorname{diam}(G)}$. Then for any $t \geq 1, \pi(G, t)=\pi_{t}(G)=$ $2^{\operatorname{diam}(G)} t$. In particular, Conjecture 3.1 holds for complete graphs, even cycles, and hypercubes.

Proof: By Lemma 3.10, $2^{\operatorname{diam}(G)} t \leq \pi_{t}(G)$. Conversely, given $2^{\operatorname{diam}(G)} t$ pebbles, we can split them into $t$ groups of $2^{\text {diam }(G)}$ pebbles each. Then each group can be matched to a different pebble in any target distribution with $t$ pebbles. Thus, $2^{\operatorname{diam}(G)} t \leq \pi_{t}(G) \leq \pi(G, t) \leq 2^{\operatorname{diam}(G)} t$, so $\pi_{t}(G)=\pi(G, t)$.

Theorem 3.5 If $G$ is a tree or a cycle, then $\pi(G, t)=\pi_{t}(G)$.

Proof: If $G$ is a tree, by Theorem 2.1 and Proposition 2.2 , the cost of putting a pebble on any vertex is at most $2^{\operatorname{diam}(G)}=\pi_{t+1}(G)-\pi_{t}(G)$. If $G$ is an even cycle we can apply Theorem 3.4, so suppose $G=C_{n}$ is an odd cycle with vertices $\left\{x_{1}, x_{2}, \ldots, x_{n}\right\}$ in order with $n=2 k+1$. We may assume 
without loss of generality that $x_{n}$ is the target vertex. By Proposition $2.3, \pi_{t}\left(C_{n}\right)$ is given by $\pi_{t}\left(C_{n}\right)=$ $\frac{2^{k+2}-(-1)^{k}}{3}+2^{k}(t-1)$. Thus, $\pi_{t+1}\left(C_{n}\right)-\pi_{t}\left(C_{n}\right)=2^{k}$, and $\pi_{t}(G) \geq 2^{k+1}$ when $t \geq 2$. In particular, if we have $\pi_{t+1}(G) \geq 2^{k+1}$ pebbles on $C_{n}$, either we have $2^{k}$ pebbles on the vertices $\left\{x_{n}, x_{1}, x_{2}, \ldots, x_{k}\right\}$ or we have $2^{k}$ pebbles on the vertices $\left\{x_{n}, x_{n-1}, x_{n-2}, \ldots, x_{k+1}\right\}$. Since these vertex sets each induce the subgraph $P_{k+1}$, we can move a pebble to $x_{n}$ at a cost of at most $\pi\left(P_{k+1}\right)=2^{k}$ pebbles.

\subsection{Fractional Pebbling Numbers}

One might wonder how the $t$-pebbling number of a graph grows with $t$. We note that for complete graphs, trees, cycles, and indeed for all other graphs $G$ for which $\pi_{t}(G)$ is known, we have $\pi_{t+1}(G) \leq$ $\pi_{t}(G)+2^{\operatorname{diam}(G)}$ for all $t$. We raise this observation to the status of a conjecture, and we prove it for large enough $t$. Conjecture 3.7 is a weaker version of Conjecture 3.6 ,

Conjecture 3.6 For every graph $G$ and for every $t \geq 1$, we have $\pi_{t+1}(G) \leq \pi_{t}(G)+2^{\operatorname{diam}(G)}$.

Conjecture 3.7 For every graph $G$ and for every $t \geq 1$, we have $\pi_{t}(G) \leq \pi(G)+2^{\operatorname{diam}(G)}(t-1)$.

Theorem 2.7 proves Conjecture 3.7 for all graphs with diameter 2. Combining Conjecture 3.7 with Theorem 2.17gives us Conjecture 3.8, and combining it with Conjecture 2.19 gives Conjecture 3.9 .

Conjecture 3.8 If $G$ is a graph with diameter 3 , then $\pi_{t}(G) \leq 1.5 n+8 t-6$.

Conjecture 3.9 If $G$ is a graph with diameter $d$, then $\pi_{t}(G) \leq\left(\frac{2^{\left\lceil\frac{d}{2}\right\rceil}-1}{\left\lceil\frac{d}{2}\right\rceil}\right) n+2^{d}(t-1)+f(d)$, for some function $f$ that depends on d only.

We show Conjecture 3.6 holds for sufficiently large $t$ after giving one lemma.

Lemma 3.10 For every graph $G$ and every integer $t \geq 1$, we have $\pi_{t}(G) \geq 2^{\operatorname{diam}(G)} t$.

Proof: We simply note that placing $2^{\operatorname{diam}(G)} t-1$ pebbles on some vertex $v$ would create a situation from which we could not move $t$ pebbles onto another vertex whose distance from $v$ is $\operatorname{diam}(G)$. 
Theorem 3.11 For every graph $G$ with $n$ vertices, and for every $t \geq\left\lceil\frac{n-1}{\operatorname{diam}(G)}\right\rceil$, we have $\pi_{t+1}(G) \leq \pi_{t}(G)+$ $2^{\operatorname{diam}(G)}$.

Proof: We let $d=\operatorname{diam}(G)$. By Lemma 3.10 we have $\pi_{t}(G) \geq 2^{d} t$, so

$$
\pi_{t}(G)+2^{d} \geq 2^{d}(t+1) \geq 2^{d}\left(\frac{n-1}{d}+1\right)=\frac{2^{d}}{d}(n-1)+2^{d} \geq \frac{2^{d}-1}{d}(n-1)+1 .
$$

Therefore, by Theorem 2.4 if we have $\pi_{t}(G)+2^{d}$ pebbles on $G$, putting the first pebble on any target vertex costs at most $2^{d}$ pebbles, so we can use the remaining $\pi_{t}(G)$ pebbles to put $t$ additional pebbles on the target.

In keeping consistent with the definitions of fractional analogues of other graph invariants, the fractional pebbling number was defined in [9] as follows.

Definition (Hurlbert [9]): The fractional pebbling number $\hat{\pi}(G)$ is given by $\hat{\pi}(G)=\liminf _{t \rightarrow \infty} \frac{\pi_{t}(G)}{t}$. In [13], we find a similar form for the definitions of the fractional analogues of chromatic number, clique number, matching number, and others. We use Theorem 3.11 to prove that $\hat{\pi}(G)=2^{\operatorname{diam}(G)}$ for every graph $G$, as conjectured in [9].

Theorem 3.12 For every graph $G$, we have $\hat{\pi}(G)=2^{\text {diam }(G)}$.

Proof: We let $s=\left\lceil\frac{n-1}{\operatorname{diam}(G)}\right\rceil$. Applying Theorem 3.11 inductively on $t$ gives $\pi_{t}(G) \leq \pi_{s}(G)+(t-$ $s) 2^{\operatorname{diam}(G)}$ for all $t \geq s$. Given $\epsilon>0$, we let $x=\pi_{s}(G)-2^{\operatorname{diam}(G)} s \geq 0$. Then for any $t \geq \max \left(\frac{x}{\epsilon}, s\right)$ we have

$$
2^{\operatorname{diam}(G)} t \leq \pi_{t}(G) \leq \pi_{s}(G)+(t-s) 2^{\operatorname{diam}(G)}=2^{\operatorname{diam}(G)} t+x
$$

Dividing by $t$ gives

$$
2^{\operatorname{diam}(G)} \leq \frac{\pi_{t}(G)}{t} \leq 2^{\operatorname{diam}(G)}+\frac{x}{t} \leq 2^{\operatorname{diam}(G)}+\epsilon .
$$

Thus, $\hat{\pi}(G)=\liminf _{t \rightarrow \infty} \frac{\pi_{t}(G)}{t}=2^{\operatorname{diam}(G)}$. 


\subsection{Optimal Fractional Pebbling Numbers}

In this section, we see that optimal pebbling can be modeled nicely as an optimization problem. This in turn leads to a nice combinatorial interpretation of the optimal fractional pebbling number of a graph. We use this interpretation to obtain a resulting property of vertex-transitive graphs. We begin by giving the generalization of a distribution to allow non-integral amounts of pebbles to be placed on each vertex.

Definition (Moews [10]): For a graph $G$, a function $D: V \rightarrow \mathbb{R}^{\geq 0}$ is called a continuous distribution on $G$. As in an integer-valued distribution, the size of $D$ is given by $|D|=\sum_{v \in V} D(v)$.

We give the following definition, which serves to generalize the notion of a pebbling move.

Definition (Moews [10]): A continuous pebbling move of size $\alpha \in \mathbb{R}^{+}$from a vertex $v$, which has at least $2 \alpha$ pebbles, to a vertex $u \in N(v)$ removes $2 \alpha$ pebbles from $v$ and places $\alpha$ pebbles on $u$.

Thus, the pebbles are no longer discrete objects. Instead, they can be viewed as infinitely divisible "piles". Nevertheless, for a vertex $v$, a continuous distribution $D$, and a nonnegative real number $\alpha$, if $D(v)=\alpha$, then we say that there are $\alpha$ pebbles on $v$ under $D$.

Definition: A continuous distribution $D$ on a graph $G$ is called optimal if the following two conditions hold.

1. For every $v \in V$, one pebble can be moved to $v$ after some sequence of continuous pebbling moves, starting from $D$.

2. If $D^{\prime}$ is a continuous distribution on $G$ with $\left|D^{\prime}\right|<|D|$, then there is some $v \in V$ which cannot be reached with one pebble after any sequence of continuous pebbling moves, starting from $D^{\prime}$.

Recall that for a graph $G$ and an integer $t \geq 1, \pi_{t}^{*}(G)$ is the size of the smallest $t$-fold solvable distribution of pebbles on $G$. Thus, given a $t$-fold solvable distribution $D$ on $G$, every $v \in V$ must have a corresponding sequence of pebbling moves that places $t$ pebbles on $v$, starting from $D$. Let 
$V=\left\{v_{1}, v_{2}, \ldots, v_{n}\right\}$. For all $i, j$, and $k$, let $p_{i, j, k}$ denote the number of pebbling moves from $v_{j}$ to $v_{k}$ in the sequence of moves which places a pebble on $v_{i}$. Let us refer to the following integer optimization problem as OPT.

The OPT Integer Optimization Problem: Minimize $\sum_{i=1}^{n} D\left(v_{i}\right)$ subject to the following constraints for each $i, j$, and $k$ with $1 \leq i, j, k \leq n$ :

$$
\begin{gathered}
D\left(v_{i}\right)+\sum_{v_{j} \sim v_{i}}\left(p_{i, j, i}-2 p_{i, i, j}\right) \geq t \\
D\left(v_{k}\right)+\sum_{v_{j} \sim v_{k}}\left(p_{i, j, k}-2 p_{i, k, j}\right) \geq 0 \\
D\left(v_{i}\right) \in \mathbb{N} \\
p_{i, j, k} \in \mathbb{N}
\end{gathered}
$$

Clearly, every $t$-fold solvable distribution $D$ on $G$ results in a feasible solution to OPT. Indeed, every pebbling move from a vertex removes two pebbles from it and every pebbling move to a vertex adds a pebble to it. Thus, after any sequence of pebbling moves which places at least $t$ pebbles on vertex $v_{i}$, every vertex must end up with a nonnegative number of pebbles while $v_{i}$ ends up with at least $t$ pebbles. Conversely, Watson [14] shows that every feasible solution to OPT results in a $t$-fold solvable distribution on $G$. Thus, the solution to OPT is equal to $\pi_{t}^{*}(G)$.

We give the following definition, which is similar to that of $\hat{\pi}(G)$.

Definition: The optimal fractional pebbling number $\hat{\pi}^{*}(G)$ is given by $\hat{\pi}^{*}(G)=\liminf _{t \rightarrow \infty} \frac{\pi_{t}^{*}(G)}{t}$.

Suppose that we desire a combinatorial interpretation for $\hat{\pi}^{*}(G)$. In this spirit, suppose we relax the integer constraints in OPT and set $t=1$. Let us refer to the following optimization problem as FRAC OPT. We denote its solution ofc $(G)$, as in [10], where this quantity is referred to as the continuous optimal pebbling number of $G$.

The FRAC OPT Optimization Problem: Minimize $\sum_{i=1}^{n} D\left(v_{i}\right)$ subject to the following constraints for 
each $i, j$, and $k$ with $1 \leq i, j, k \leq n$ :

$$
\begin{gathered}
D\left(v_{i}\right)+\sum_{v_{j} \sim v_{i}}\left(p_{i, j, i}-2 p_{i, i, j}\right) \geq 1 \\
D\left(v_{k}\right)+\sum_{v_{j} \sim v_{k}}\left(p_{i, j, k}-2 p_{i, k, j}\right) \geq 0 \\
D\left(v_{i}\right) \geq 0 \\
p_{i, j, k} \geq 0
\end{gathered}
$$

We show that ofc $(G)$ is equal to the optimal fractional pebbling number of $G$.

Fact 3.13 For every graph $G$, ofc $(G)=\hat{\pi}^{*}(G)$.

Proof: Let $G$ be a graph, with $V=\left\{v_{1}, v_{2}, \ldots, v_{n}\right\}$. We first show ofc $(G) \leq \hat{\pi}^{*}(G)$. For an integer $t \geq 1$, let $D$ be a $t$-fold solvable distribution on $G$ with $|D|=\pi_{t}^{*}(G)$. Then, for every $v_{i} \in V$, there are $D\left(v_{i}\right)$ pebbles initially on $v_{i}$ and there is some sequence of pebbling moves which places $t$ pebbles on $v_{i}$. This gives a solution to OPT. In this solution, let $p_{i, j, k}$ be defined as above. Now, let $D^{\prime}\left(v_{i}\right)=\frac{D\left(v_{i}\right)}{t}$ and let $p_{i, j, k}^{\prime}=\frac{p_{i, j, k}}{t}$ for all $i, j$, and $k$. This gives a feasible solution to FRAC OPT with $\left|D^{\prime}\right|=\frac{\pi_{t}^{*}(G)}{t}$. This solution may or may not be optimal. Since this holds for any integer $t \geq 1$, we have ofc $(G) \leq \hat{\pi}^{*}(G)$.

We now show that ofc $(G) \geq \hat{\pi}^{*}(G)$. Suppose we have a feasible solution to FRAC OPT, with values denoted $\bar{D}\left(v_{i}\right)$ and $\bar{p}_{i, j, k}$ for all $i, j$, and $k$. We may assume that every $\bar{D}\left(v_{i}\right)$ and $\bar{p}_{i, j, k}$ is rational, since all of the coefficients are integers. Let $t$ be the least common multiple of the denominators of these values. Let $D^{\prime}\left(v_{i}\right)=t \bar{D}\left(v_{i}\right)$ and let $p_{i, j, k}^{\prime}=t \bar{p}_{i, j, k}$ for all $i, j$, and $k$. This gives a feasible solution to OPT and thus a $t$-fold solvable distribution $D^{\prime}$ on $G$. Clearly, $\frac{\left|D^{\prime}\right|}{t}$ is the value of the rational solution we were given. However, $D^{\prime}$ may not be the smallest $t$-fold solvable distribution on $G$. Furthermore, we can let $D^{\prime \prime}\left(v_{i}\right)=t s \bar{D}\left(v_{i}\right)$ and let $p_{i, j, k}^{\prime \prime}=t s \bar{p}_{i, j, k}$ for all $i, j$, and $k$ for any positive integer $s$ to obtain a $t s$-fold solvable distribution on $G$. Thus, ofc $(G) \geq \hat{\pi}^{*}(G)$.

The following corollary provides a combinatorial interpretation for $\hat{\pi}^{*}(G)$.

Corollary 3.14 The size of an optimal continuous distribution on a graph $G$ is equal to $\hat{\pi}^{*}(G)$. 
Proof: From the definition, we see that the size of an optimal continuous distribution on a graph $G$ is equal to the solution to the optimization problem FRAC OPT. The result follows from Fact 3.13 .

In Theorem 3.18, we show that every vertex-transitive graph has an optimal continuous distribution which is uniform. We start with some lemmas, beginning with the following self-evident weight argument.

Lemma 3.15 Let $D$ be a continuous distribution on a graph $G$. Then there is a sequence of continuous pebbling moves starting from $D$ which places a pebble on $\mathbf{r} \in V$ if and only if $\sum_{v \in V} D(v) 2^{-\operatorname{dist}(v, \mathbf{r})} \geq 1$.

The following lemma is obvious, but useful.

Lemma 3.16 If $G=(V, E)$ is a vertex-transitive graph, then the function $f: V \rightarrow \mathbb{R}^{+}$given by $f(u)=$ $\sum_{v \in V} 2^{-\operatorname{dist}(v, u)}$ is constant for all $u$.

Lemma 3.17 If $D$ and $D^{\prime}$ are continuous distributions on a vertex-transitive graph $G$ and

$$
\sum_{u \in V} D(u) 2^{-\operatorname{dist}(v, u)} \leq \sum_{u \in V} D^{\prime}(u) 2^{-\operatorname{dist}(v, u)}
$$

for all $v \in V$, then $|D| \leq\left|D^{\prime}\right|$.

Proof: Let $G=(V, E)$ be a vertex-transitive graph. Summing both sides of (5) over all $v \in V$, we find

$$
\sum_{v \in V} \sum_{u \in V} D(u) 2^{-\operatorname{dist}(v, u)} \leq \sum_{v \in V} \sum_{u \in V} D^{\prime}(u) 2^{-\operatorname{dist}(v, u)}
$$

Switching the order of the summation gives us

$$
\sum_{u \in V} D(u) \sum_{v \in V} 2^{-\operatorname{dist}(v, u)} \leq \sum_{u \in V} D^{\prime}(u) \sum_{v \in V} 2^{-\operatorname{dist}(v, u)}
$$

But by Lemma 3.16, $\sum_{v \in V} 2^{-\operatorname{dist}(v, u)}$ is a constant for all $u \in V$, so dividing by this constant gives us $\sum_{u \in V} D(u) \leq \sum_{u \in V} D^{\prime}(u)$, or $|D|<\left|D^{\prime}\right|$

We are now ready to show the main result for this section. 
Theorem 3.18 If $G$ is a vertex-transitive graph, an optimal continuous distribution on $G$ is obtained by putting $\frac{1}{m}$ pebbles on each vertex in $G$, where $m$ is the constant $\sum_{v \in V} 2^{-\operatorname{dist}(v, u)}$ from Lemma 3.16 Therefore, $\hat{\pi}^{*}(G)=\frac{n}{m}$.

Proof: Let $D$ be the distribution in question. Note that for any $\operatorname{root} \mathbf{r} \in V$, we have

$$
\sum_{v \in V} D(v) 2^{-\operatorname{dist}(v, \mathbf{r})}=\frac{1}{m} \sum_{v \in V} 2^{-\operatorname{dist}(v, \mathbf{r})}=1,
$$

so by Lemma 3.15, starting from $D$, the root $\mathbf{r}$ can receive a pebble by making continuous pebbling moves toward $\mathbf{r}$. Therefore, $\hat{\pi}^{*}(G) \leq|D|$.

Now let $D^{\prime}$ be another continuous distribution from which one pebble can be moved to $\mathbf{r}$. By Lemma 3.15, we have

$$
\sum_{v \in V} D^{\prime}(v) 2^{-\operatorname{dist}(v, \mathbf{r})} \geq 1=\sum_{v \in V} D(v) 2^{-\operatorname{dist}(v, \mathbf{r})}
$$

for all $v \in V$, and by Lemma 3.17, this implies $\left|D^{\prime}\right| \geq|D|$. Therefore, $D$ is optimal, so $\hat{\pi}^{*}(G)=|D|$.

Corollary 3.19 gives $\hat{\pi}^{*}(G)$ for several vertex-transitive graphs. Moews [10] also proved part1.

Corollary 3.19 Let $k$ and $n$ be positive integers. Then we have the following.

1. $\hat{\pi}^{*}\left(Q^{k}\right)=\left(\frac{4}{3}\right)^{k}$ where $Q^{k}$ denotes the $k$-dimensional hypercube.

2. $\hat{\pi}^{*}\left(K_{n}\right)=\frac{2 n}{n+1}$.

3. If $k \geq 2$, then $\hat{\pi}^{*}\left(C_{2 k}\right)=\frac{k 2^{k+1}}{3\left(2^{k}-1\right)}$.

4. $\hat{\pi}^{*}\left(C_{2 k+1}\right)=\frac{(2 k+1)\left(2^{k-1}\right)}{3\left(2^{k-1}\right)-1}$.

Proof: By Theorem 3.18 , in each case it suffices to find the value of $m$. For the hypercube, if we fix a target $\mathbf{r}$, there are $\left(\begin{array}{c}k \\ i\end{array}\right)$ vertices whose distance from $\mathbf{r}$ is $i$. We compute $m$ as follows, using the Binomial Theorem.

$$
m=\sum_{v \in V} 2^{-\operatorname{dist}(v, \mathbf{r})}=\sum_{i=1}^{k}\left(\begin{array}{l}
k \\
i
\end{array}\right) \frac{1}{2^{i}}=\left(\frac{3}{2}\right)^{k} .
$$


Therefore, $\hat{\pi}^{*}\left(Q^{k}\right)=\frac{n}{m}=\frac{2^{k}}{\left(\frac{3}{2}\right)^{k}}=\left(\frac{4}{3}\right)^{k}$.

For $K_{n}$ every vertex $v \neq r$ has $\operatorname{dist}(v, \mathbf{r})=1$, so

$$
m=1+\sum_{v \in V ; v \neq \mathbf{r}} \frac{1}{2}=1+\frac{n-1}{2}=\frac{n+1}{2}
$$

and $\hat{\pi}^{*}\left(K_{n}\right)=\frac{n}{\frac{n+1}{2}}=\frac{2 n}{n+1}$.

For $C_{n}$ we assume the vertex set is $\left\{x_{0}, x_{1}, \ldots, x_{n-1}\right\}$ and that $\mathbf{r}=x_{0}$ is the target. If $n=2 k$, we let $A=\left\{x_{i}: i<k\right\}$, and we note that for every $x_{k+i}$ with $0 \leq i \leq k-1$ we have $\operatorname{dist}\left(x_{k+i}, x_{0}\right)=k-i$. Therefore, computing $m$ gives

$$
m=\sum_{v \in V} 2^{-\operatorname{dist}\left(v, x_{0}\right)}=\sum_{v \in A} 2^{-\operatorname{dist}\left(v, x_{0}\right)}+\sum_{v \notin A} 2^{-\operatorname{dist}\left(v, x_{0}\right)}=\sum_{i=0}^{k-1} 2^{-i}+\sum_{i=0}^{k-1} 2^{-(k-i)} .
$$

Substituting $j=k-1-i$ in the last summation gives

$$
m=\sum_{i=0}^{k-1} 2^{-i}+\sum_{j=0}^{k-1} 2^{-(j+1)}=\sum_{i=0}^{k-1} 2^{-i}+\frac{1}{2} \sum_{j=0}^{k-1} 2^{-j}=\frac{3}{2}\left(2-\frac{1}{2^{k-1}}\right)=\frac{3\left(2^{k}-1\right)}{2^{k}} .
$$

Therefore, $\hat{\pi}^{*}\left(C_{2 k}\right)=\frac{n}{m}=\frac{2 k\left(2^{k}\right)}{3\left(2^{k}-1\right)}=\frac{k 2^{k+1}}{3\left(2^{k}-1\right)}$.

Finally, for $C_{2 k+1}$ we let $A=\left\{x_{i}: 1 \leq i \leq k\right\}$ and $B=\left\{x_{i}: k+1 \leq i \leq 2 k\right\}$. Now $\operatorname{dist}\left(x_{k+i}, x_{0}\right)=$ $k-i+1$, so we have

$$
m=\sum_{v \in V} 2^{-\operatorname{dist}\left(v, x_{0}\right)}=2^{-\operatorname{dist}\left(x_{0}, x_{0}\right)}+\sum_{v \in A} 2^{-\operatorname{dist}\left(v, x_{0}\right)}+\sum_{v \in B} 2^{-\operatorname{dist}\left(v, x_{0}\right)}=1+\sum_{i=1}^{k} 2^{-i}+\sum_{i=1}^{k} 2^{-(k-i+1)} .
$$

Now substituting $j=k-i+1$ gives

$$
m=1+\sum_{i=1}^{k} 2^{-i}+\sum_{j=1}^{k} 2^{-j}=1+2 \sum_{i=1}^{k} 2^{-i}=1+2\left(1-\frac{1}{2^{k}}\right)=3-\frac{1}{2^{k-1}}=\frac{3\left(2^{k-1}\right)-1}{2^{k-1}} .
$$

Therefore, $\hat{\pi}^{*}\left(C_{2 k+1}\right)=\frac{n}{m}=\frac{(2 k+1)\left(2^{k-1}\right)}{3\left(2^{k-1}\right)-1}$.

\section{References}

[1] A. Bekmetjev and C. Cusack, Pebbling algorithms in diameter two graphs, SIAM J. Discrete Math. 23 (2009), No. 2, 634-646. 
[2] A. Blasiak and J. Schmitt, Degree sum conditions in graph pebbling, Australas. J. Combin. 42 (2008), 83-90. Pebbling in diameter two graphs and products of paths, J. Graph Theory 25 (1997), 119-128.

[3] B. Bukh, Maximum pebbling numbers of graphs of diameter three, J. Graph Theory 52 (2006), 353-357.

[4] D. P. Bunde, E. W. Chambers, D. Cranston, K. Milans, and D. B. West, Pebbling and optimal pebbling in graphs, J. Graph Theory 57 (2008), 215-238.

[5] F. R. K. Chung, Pebbling in hypercubes, SIAM J. Discrete Math. 2 (1989), No. 4, 467-472.

[6] T.A. Clarke, R.A. Hochberg, and G.H. Hurlbert, Pebbling in diameter two graphs and products of paths, J. Graph Theory 25 (1997), 119-128.

[7] D. Curtis, T. Hines, G. Hurlbert, and T. Moyer, On pebbling graphs by their blocks, Integers: Elec. J. Number Th. 9 (2009), \#G2, 411-422.

[8] D. S. Herscovici, Graham's pebbling conjecture on products of cycles, J. Graph Theory 42 (2003), No. 2, 141-154.

[9] G. Hurlbert, The graph pebbling page, http://mingus.la.asu.edu/ hurlbert/pebbling/pebb.html.

[10] D. Moews, Optimally pebbling hypercubes and powers, Discrete Mathematics 190 (1998), 271276.

[11] L. Pachter, H. S. Snevily, and B. Voxman, On pebbling graphs, Congr. Numer. 107 (1995), 65-80.

[12] L. Postle, N. Streib, and C. Yerger, Pebbling graphs of diameter three and four, European Conference on Combinatorics, Graph Theory and Applications (EuroComb 2009), 21-28, Electron. Notes Discrete Math. 34, Elsevier Sci. B. V., Amsterdam, 2009. 
[13] E.R. Scheinerman and D.H. Ullman, Fractional graph theory: A rational approach to the theory of graphs, John Wiley \& Sons, Inc., New York, 1997.

[14] N. Watson, The complexity of pebbling and cover pebbling, http://arXiv.org/abs/math.CO/0503511 (2005). 